\title{
The phyllotaxis of the aortic valve
}

\author{
Marco Moscarelli ${ }^{1,2}$, Ruggero De Paulis ${ }^{3}$ \\ ${ }^{1}$ Imperial College, National Heart and Lung Institute, London, UK; ${ }^{2}$ GVM Care \& Research, Cotignola, Italy; \\ ${ }^{3}$ Department of Cardiac Surgery, European Hospital, Rome, Italy
}

\section{Introduction}

We congratulate Koshkelashvili and colleagues for their very interesting and original study on aortic root and valve proportion [1]. They scrutinized 150 patients undergoing ECG gated cardiac CT scan for different clinical indications and performed 3D reconstruction. The Authors' conclusions can be summarized as follow: there was an expected gender variability; there were some differences in leaflets area; it also seemed that the right Valsalva expanded more in systole. Perhaps, the most interesting finding of this study was that the observed ratio of sinus height / annular radio was ' 1.69 ', that was very close to the so-called 'golden ratio', a number that is often encountered in nature.

As cardiac surgeons, by observing the fascinating geometry of a normal tricuspid aortic valve, we were previously able to speculate that spirals with such intrinsic ' 1.6 ratio' would perfectly fit into the aortic valve, representing an excellent way to describe its unique symmetry [2] (Figure 1). In our study, differently from the authors, we analyzed the trans-esophageal short axis view of various tricuspid aortic valves.

With this editorial we would like to shed lights on the 'golden-ratio' while trying to clarify its role in the modern science.

Correspondence: Marco Moscarelli, Anthea Hospital, Via Camillo Rosalba 35/37, 70124 Bari, Italy.

Tel. +39.080.5644111 - Fax: +39.080.5644678.

E-mail: m.moscarelli@imperial.ac.uk

Key words: aortic valve; golden ratio; golden spiral; Fibonacci; phyllotaxis.

Disclosure: none.

Authors' contribution: The authors contribute equally to the writing of paper.

Received for publication: 29 July 2019.

Accepted for publication: 30 July 2019.

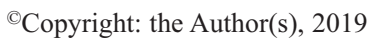

Licensee PAGEPress, Italy

Monaldi Archives for Chest Disease 2019; 89:1139

doi: 10.4081/monaldi.2019.1139

This article is distributed under the terms of the Creative Commons Attribution Noncommercial License (by-nc 4.0) which permits any noncommercial use, distribution, and reproduction in any medium, provided the original author(s) and source are credited.

\section{The science of the 'golden ratio' in cardiac anatomy}

Some physicians claim that there are specific shapes and structures in the human body that might respect the 'golden-ratio'. However, the question is: does this ratio have any rational and scientific validity? Is it just a number identified by chance? Ashrafian et al. suggested that the coronary arterial branching system has strict analogy with the branching pattern of a tree, and that the Fibonacci series might represent a novel bio-mathematical model of coronary arterial development [3]. Thuroff et al. also supposed that the ratio of the sum of the diameters of the major coronary branches is close to the 'golden-ratio' [4]. Henein et al. [5] analyzed the mitral valve of both healthy and patients with functional mitral regurgitation and dilated cardiomyopathy; they realized that the ratio (length/width) of the mitral annulus in the normal group and in patients with dilated cardiomyopathy was 1.62 and 1.42 respectively. They concluded that deviation from the normal anatomy represented by the 'golden-ratio', might lead to distortion and enlargement of the annulus, hence to mitral regurgitation. Interestingly, as cardiac surgeons, we also observed that one of the most common prosthetic mitral ring (Memo 3D Livanova) that is used to reshape the native mitral annulus has also a ratio length/width of 1.6 as shown in Figure 2.

Great arteries may arise from the heart with an inclination close to the 'golden-angle' [5].

There are also several echocardiographic, electrocardiographic and functional indexes that have been described to conform to the golden proportion [6]. Lastly, we have previously hypothesized that the normal tricuspid aortic valve may be described, in its two-dimensional shape, as an approximation of golden spirals, that ultimately may have several similarities to the fractal geometry observed in plants [2].

\section{The history of the 'golden-ratio'}

The basis of the golden proportion $(a / b=\varphi=1.6)$ can be found in the ancient Greek architecture [7]. This ratio was described by Euclid as 'extreme and mean ratio' [8]. During the $13^{\text {th }}$ century, the Italian mathematician Leonardo Bonacci (better known as Fibonacci), described in the Liber Abaci the famous 'Fibonacci sequence' $(1,1,2,3,5,8 \ldots)$, in which the ratio of every consecutive number approximates the value of '1.618'. Nonetheless, the Fibonacci sequence was recognized earlier by the Indian mathematician Pingala, who has received far less reputation as compared to Fibonacci [9].

Da Vinci portrayed the Vitruvian man (ca. 1490) and such sketch represents with its 'divine proportion' the symbol of the Renaissance, of the equilibrium of man and mankind [10]. 
It is however worth to notice that the term 'golden-ratio' was only coined by Martin Ohm (1792-1872) in his book 'The Pure Elementary Mathematics' and the term 'phi' was not used until the 1900 when the American mathematician Mark Burr used the Greek letter to designate this proportion.

It is from the Fibonacci sequence that is possible to generate the 'Fibonacci spiral' that overlaps almost perfectly the 'golden spiral'. Such spiral structures are worldwide present in pinecones, shells, sunflowers etc. The Fibonacci sequence is also at the base of the 'phyllotaxis', that is the part of the botanic science that deals with the arrangements and branching of plants. Whether this rule could be applied to humans, however, is still under investigation.

\section{A lesson from the plants}

While physicians might be not very familiar with the goldenratio, botanists are. The word phyllotaxis derives from the Greek phyllon (leaf) and taxis (arrangement), and deals with the arrangements of plant organs such as leaves, bracts, branches, petals, florets and scales, called in their younger stage 'primordia'. It is accepted
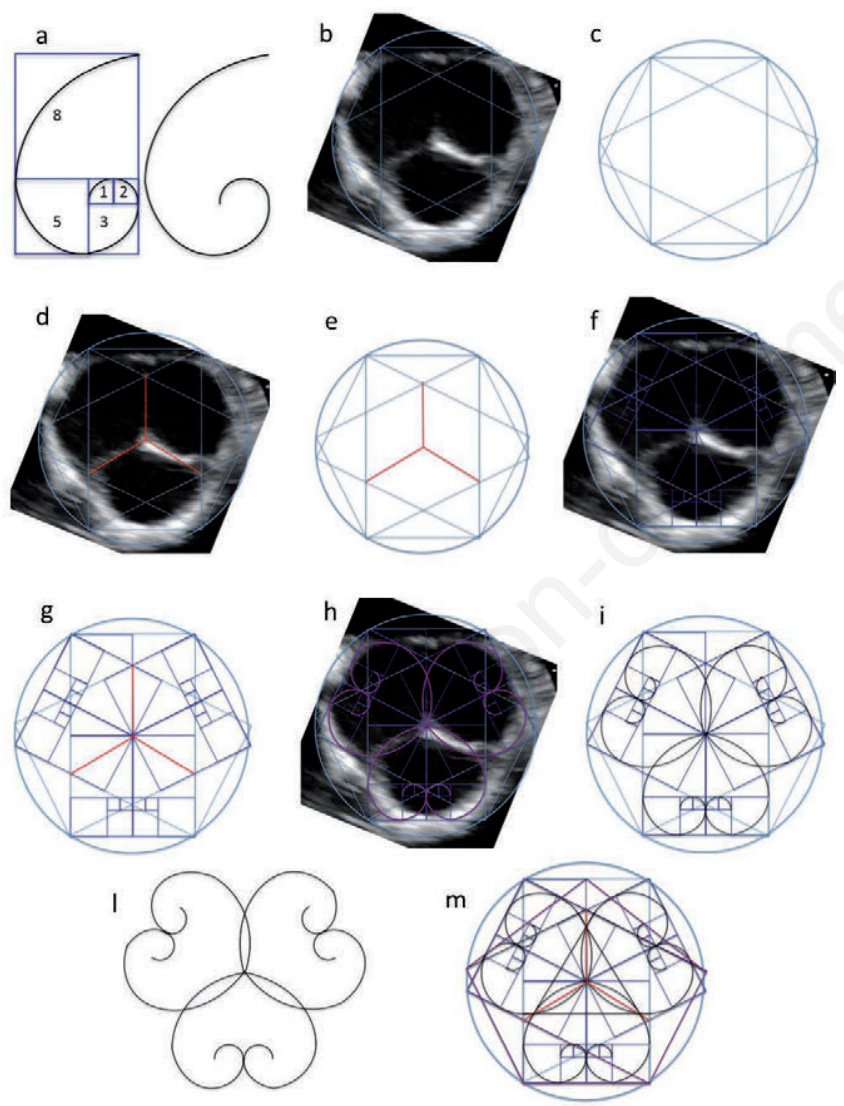

Figure 1. 'The golden perfection of the aortic valve'. A Fibonacci spiral approximates the golden spiral using quarter-circle arcs inscribed in squares of integer Fibonacci-number side, shown for square sizes 1, 2, 3, 5, 8 (panel a). The figure shows the fascinating symmetry of the aortic valve that ultimately can be represented by six golden spirals inscribed in regular pentagons that may be cut into squares and smaller rectangles with the same aspect ratio. From: Moscarelli M, De Paulis R. Int J Cardiol 2016;205:165-6; reproduced with permission. that the first report of phyllotaxis goes back to Theophrastus (370 B.C. \pm 285 B.C.) and Pliny (23 A.D. \pm 79 A.D.). However, we observed that in the 'tomb of the diver' (470 B.C.) discovered in Paestum (Italy) (http://www.museopaestum.beniculturali.it/latomba-del-tuffatore), a plant with branches following a Fibonacci series $(1,1,2,3,5)$ and 'opposite phyllotaxis leaves' is pictured in details. It is therefore possible that the human interest in phyllotaxis might have started earlier than that (Figure 3).

In the various areas of botany, phyllotaxis is often considered to be the most striking phenomenon yet paradoxically simple as all the phyllotactic patterns can be described with few mathematical constants. Moreover, all the phyllotactic systems show spirality that belong to the Fibonacci type of integers, characterized by the rule that every term is the sum of the preceding two terms as in the Fibonacci sequence.

\section{A lesson with Da Vinci}

Why should we care for the shape of things? As we now know, plants branch in a non-chaotic way in order to reduce stress and

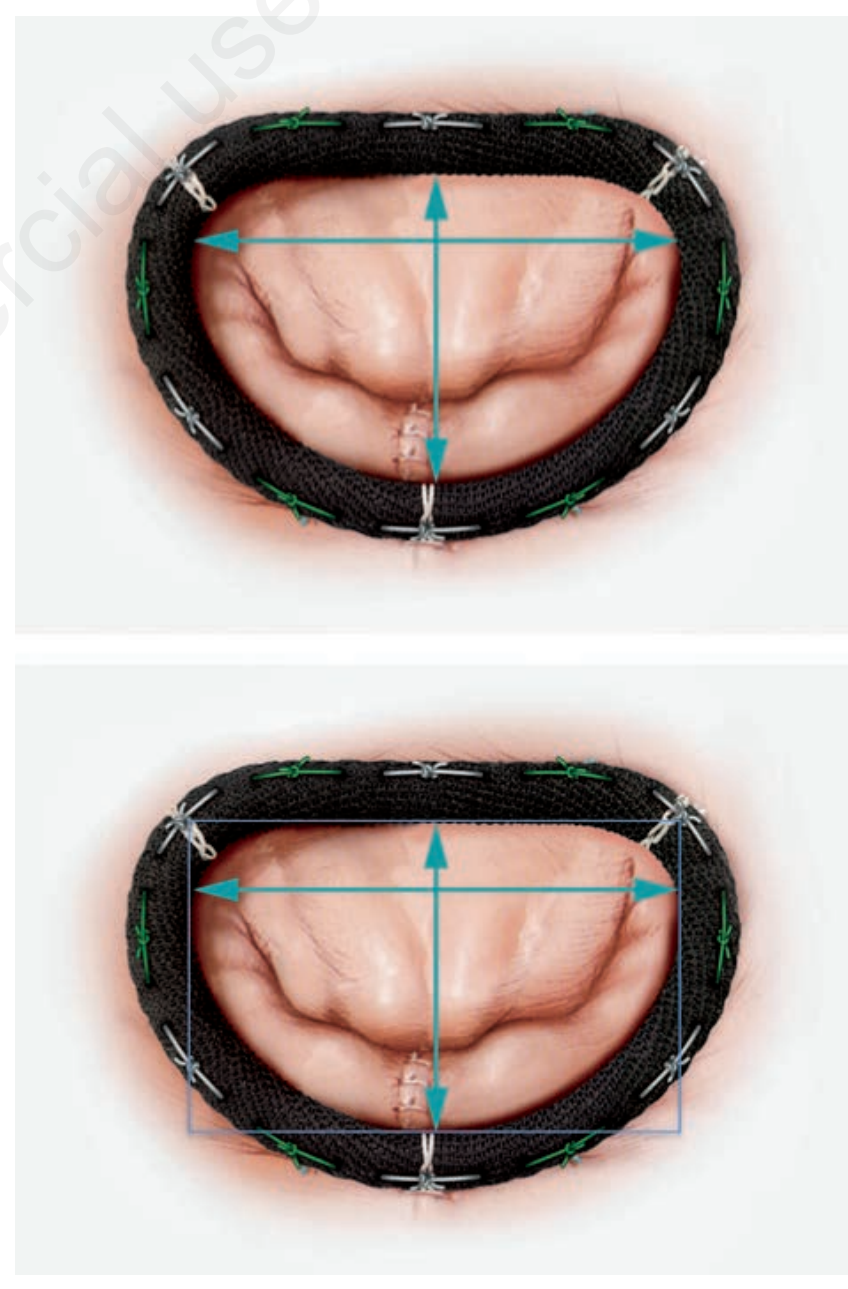

Figure 2. Prosthetic mitral-ring that is used in cardiac surgery to reshape the annulus of an enlarged mitral valve. The ratio length/width of the prosthesis yields to 1.6, the so-called 'golden-ratio'. 
maximize exposure to the sun. It is called 'adaptive mechanism or adaptive value'. Most of the plants follow the Fibonacci sequence with the 'golden-ratio' and 'golden-angles', making spectacular fractals and spirals: this is the case of pinecones, sunflowers, Romanesco broccoli among many other. In the essence, plants exhibit a functional and non-stochastic shape. While this phyllotactic rules can be observed also in humans, it is less clear whether it could also apply in the human body.

Nevertheless, the aortic valve is a striking example of human fractal geometry.

In 1510, Leonardo Da Vinci was granted from the church authorities the permission to perform cadaveric dissection. He worked deeply on the anatomy of the aortic valve and of the sinuses of Valsalva with the eddy currents. In the 'folio 115 verso of the Corpus of the Anatomical Studies' he described the perfect symmetries of the normal tricuspid valves, identifying abnormal variant such as bicuspid and quadricuspid aortic valves. He was able to understand that such deviation from the normal fractal anatomy could lead to turbulent blood flow and eventually to valve dysfunction [11]. He clearly explained that a square valve ('the quadricuspid valve') has larger orifice than a tricuspid one. However, the leaflets of a quadricuspid valve are weaker than those of a tricuspid
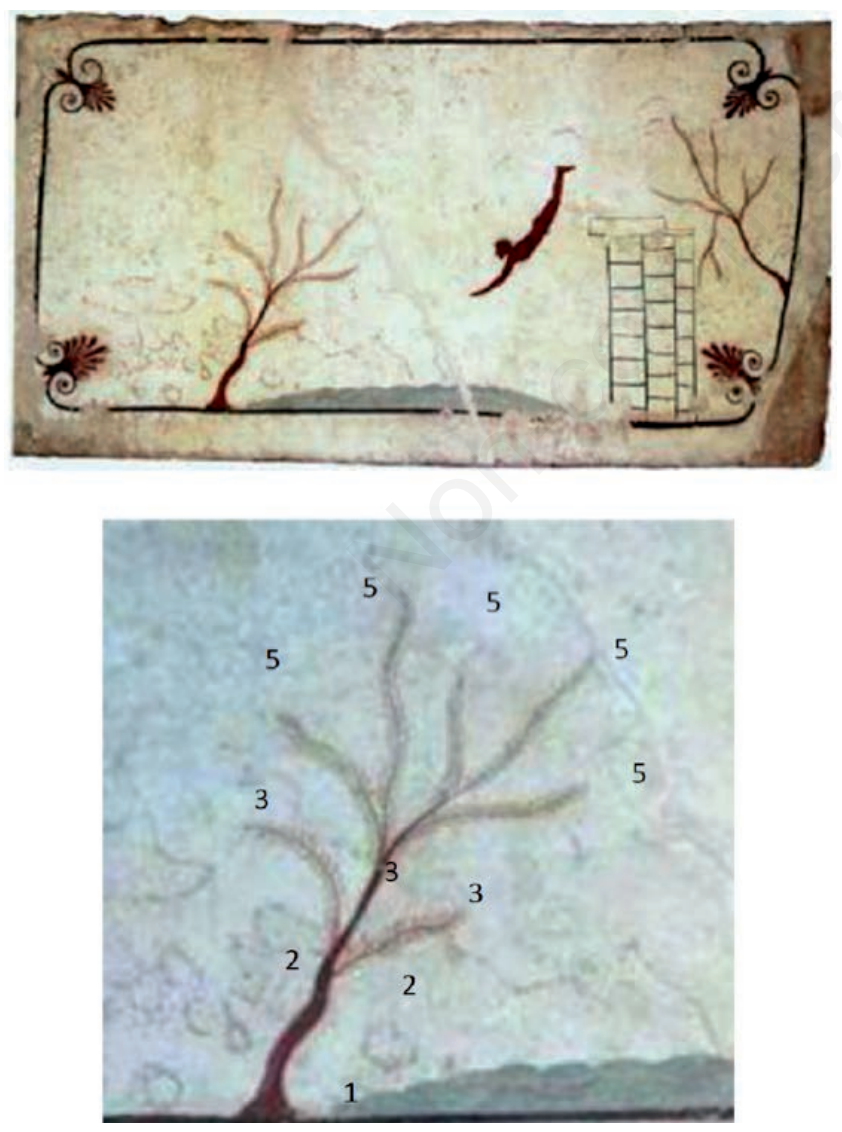

Figure 3. The Tomb of the Diver $(480 / 70 \mathrm{BC})$ is the only evidence for large-scale Greek painting, other than on vases, prior to the fourth century B.C. A tree with a simple 'opposite phyllotaxis leaves' is pictured in details. valve because their closing angles are more distant from the base of the triangle (basal attachment of the leaflet) that is the open configuration of the aortic valve [12].

This biophysical concept of optimal adaptation is also found in plant biology, where the golden-angle is adopted for phyllotaxis purposes to optimize light falling and stress resistance [13].

Leonardo had some rudimental understanding of phyllotaxis, and to our knowledge never mentioned Fibonacci in all of his experiments. Nevertheless his sketches of the aortic valve represent a masterpiece of anatomy, hemodynamic, and fractal geometry. As also mentioned by Koshkelashvili and colleagues, the organized and non-chaotic eddy currents he depicted at the level of Valsalva resemble in details the Fibonacci spirals.

\section{Discussion}

Biological systems ubiquitously and inevitably exhibit stochasticity in traits from the molecular level to the multicellular and morphological level. However, there are several examples of natural events that might be described in mathematical terms. Plants grow in a structured and geometric way to maximize their sun exposure for photosynthesis while reducing the stress. The 'Fibonacci sequence' and its 'golden ratio' are considered a mathematical regularity and model that is one of the corner-stone of the 'phyllotaxis', the part of the botany that studies how plants branch. Nevertheless, we currently do not know if such mathematical model can be applied to humans. Different authors have hypothesized that 'fractal' might be identified along with the 'golden-ratio' in the human body (coronary artery, heart valves, etc.). The aortic valve and the aortic root might represent an interesting model of human fractal geometry, where the phyllotactic rules can be reasonably applied, and where deviation from normality might results in dysfunction. However, in the absence of scientific validations, such report represents only the authors' perceptions of a beautiful shape.

\section{Perspective}

The detailed understanding of a normal aortic valve, root dimension and standard proportion is of paramount importance because various diseases can alter these relationships. When attempting to reconstruct a tricuspid or a bicuspid valve, and to replace the root, this knowledge becomes pivotal. To this extent, surgical valve prostheses or transcatheter aortic valve prostheses should ideally mimic the original proportion of the nature.

\section{References}

1. Koshkelashvili N, Jalife Bucay M, Goykhman I, Pressman GS. Aortic root and valve proportions: an example of the golden ratio? Monaldi Arch Chest Dis 2019;89. Doi: 10.4081/monaldi.2019.1022.

2. Moscarelli M, De Paulis R. The golden perfection of the aortic valve. Int J Cardiol 2016;205:165-6.

3. Ashrafian H, Athanasiou T. Fibonacci series and coronary anatomy. Heart Lung Circ 2011;20:483-4.

4. Thuroff JW, Hort W, Lichti H. Diameter of coronary arteries in 
36 species of mammalian from mouse to giraffe. Basic Res Cardiol 1984;79:199-206.

5. Henein MY, Golden Ratio Collaborators, Zhao Y, et al. The human heart: application of the golden ratio and angle. Int J Cardiol 2011;150:239-42.

6. Yalta K, Ozturk S, Yetkin E. Golden Ratio and the heart: A review of divine aesthetics. Int J Cardiol 2016;214:107-12.

7. Pasipoularides A. Historical continuity in the methodology of modern medical science: Leonardo leads the way. Int J Cardiol 2014;171:103-15.

8. Ozturk S, Yalta K, Yetkin E. Golden ratio: A subtle regulator in our body and cardiovascular system? Int J Cardiol 2016;223:143-145.
9. Singh P. The so-called Fibonacci numbers in ancient and medieval India. Historia Mathematica 1985:229-44.

10. Le Floch-Prigent P. [The Vitruvian Man: an anatomical drawing for proportions by Leonardo Da Vinci].[Article in French]. Morphologie 2008;92:204-9.

11. Ashrafian H, Harling L and Athanasiou T. Leonardo da Vinci and the first portrayal of quadricuspid semilunar valves and the nodules of Arantius. Int J Cardiol. 2013;165:560-1.

12. Morea M, De Paulis R. 'Il buso' (the orifice). How much did Leonardo know of the aortic valve? J Cardiovasc Med (Hagerstown) 2007;8:399-403.

13. Okabe T. Biophysical optimality of the golden angle in phyllotaxis. Sci Rep 2015;5:15358 